\title{
Características clínicas e histopatológicas del cáncer de próstata diagnosticado a partir de la tercera biopsia
}

\author{
Bahílo Mateu MP, Ramírez Backhaus M, Trassierra Villa M, Di Capua Sacoto C, Arlandis \\ Guzmán S, Jiménez Cruz JF.
}

Servicio de Urología. Hospital Universitario La Fe. Valencia.

Actas Urol Esp. 2008;32(10):961-967

\section{RESUMEN}

\section{CARACTERÍSTICAS CLÍNICAS E HISTOPATOLÓGICAS DEL CÁNCER DE PRÓSTATA DIAGNOSTICADO}

\section{A PARTIR DE LA TERCERA BIOPSIA}

Objetivo: Estudiar las características histopatológicas y clínicas de los tumores diagnosticados a partir de terceras biopsias para valorar la relevancia clínica de éstos y descartar el sobrediagnóstico en el cáncer de próstata.

Material y método: Revisamos las historias clínicas de 61 pacientes con al menos tres o más biopsias de próstata transrectales, sextantes o de saturación según el esquema preestablecido, desde el año 2000 al 2006. Las variables analizadas son: edad del paciente, PSA, cociente PSA libre/PSA total, PSA densidad, tacto rectal, volumen prostático, hallazgos ecográficos y lesión premaligna fuertemente asociadas a la presencia de tumor en biopsias previas. Y estudiamos la anatomía patológica de los tumores diagnosticados a partir de la tercera biopsia, abordaje terapéutico y su evolución con un seguimiento mínimo de 3 meses.

Resultados: De 61 pacientes con más de tres biopsias, a 15 pacientes se les diagnostica cáncer (24,6\%) en la tercera biopsia. A 14 se les realiza nueva biopsia (cuarta), encontrando tumor en 5 de ellos (35,7\%). Se realiza quinta biopsia en 2 pacientes siendo positiva en uno de ellos (50\%). Según los resultados de la biopsia, 6 pacientes presentaban criterios de cáncer clínicamente no significativo $(28,6 \%)$. En todos los pacientes se procedió a tratamiento curativo: 5 braquiterapia, 6 radioterapia externa y a 10 se les realizó una prostatectomía radical, que evidenció tumores clínicamente significativos: 2 tumores pT2b y 7 tumores pT2c sin invasión de los márgenes quirúrgicos y con un excelente control de la enfermedad tras un seguimiento mínimo de 13 meses y un pT4 por invasión del cuello vesical.

Conclusiones: En nuestro medio, la tercera, cuarta y quinta biopsia alcanza una positividad del $34,42 \%$ y se corresponde con tumores que se benefician de un tratamiento con intención curativa.

Palabras clave: Biopsia prostática de repetición. Cáncer de próstata.

\section{ABSTRACT}

CLINICAL FEATURES AND HISTOPATHOLOGY OF PROSTATE CANCER DIAGNOSED FROM THE THIRD BIOPSY

Objective: To study of histological and clinical features of prostate cancer diagnosed after three or more prostate biopsies in order to assess its clinical relevance and to discard the overdiagnosis of prostate cancer.

Material and methods: We reviewed the clinical records of 61 patients who underwent three or more prostate biopsies between January 2000 and December 2006. The analyzed variables were: age, PSA level, free/total PSA ratio, PSA density, digital rectal examination, prostate volume, sonographic findings and previous malignant lesion strongly associated to the presence of tumor on previous biopsy. We studied the pathology of the tumors diagnosed from the third biopsy, therapeutical approach and its evolution with a minimum follow-up of 3 months.

Results: Fifteen out of 61 patients with more than three biopsies had prostate cancer $(24,59 \%)$ in the third biopsy, 5 out of 14 patients with 4 biopsies (35,71\%) and 1 of the 2 cases (50\%) who underwent a fifth biopsy. According to the results of biopsy, 6 patients met the criteria of clinically insignificant cancer (28,57\%). Curative treatment was performed in all patients: brachytherapy in 5, external beam radiotherapy in 6 and radical prostatectomy in 10. Clinically significant tumors were found in all cases: 2 pT2b tumors and 7 pT2c tumors with negative surgical margins and with an excellent control of the cancer after a minimum follow up of 13 months, and one pT4 tumor with bladder neck infiltration.

Conclusion: In our practice, overall detection rate of the third, fourth and fifth biopsy is $34,42 \%$ corresponding with tumors that could benefit from curative treatment. 
$\mathrm{E}$ 1 cáncer de próstata es uno de los más frecuentes en los países occidentales. Según datos del Instituto Nacional de Estadística, en España constituye la segunda causa de defunción por tumor en varones. En el año 2006 se registraron 5.412 defunciones y la tasa de mortalidad fue de 12,28/100.000 varones de cualquier edad ${ }^{1}$.

Para el diagnóstico de esta enfermedad nos servimos del tacto rectal (TR) y el antígeno prostático específico (PSA). Este último es el mejor factor predictivo de cáncer de próstata del que se dispone hasta el momento ${ }^{2-4}$, pero aún siendo un buen marcador tumoral, dista bastante de ser el marcador ideal de cribado. Hasta la actualidad, no se ha definido el límite entre los pacientes con alto y bajo riesgo de enfermedad, ni el límite a partir del cual no hay riesgo de cáncer. En la mayoría de estudios se utiliza un nivel de PSA mayor de $4 \mathrm{ng} / \mathrm{mL}$ para la indicación de biopsia, aunque en los últimos años existe una tendencia generalizada a disminuir este valor, sobretodo en varones jóvenes entre 50 y 66 años ${ }^{5}$.

La ecografía transrectal (ETR) puede ayudar al diagnóstico de tumores no palpables y es el método de elección para realizar la biopsia de áreas intraprostáticas sospechosas. Pero, al igual que el TR y el PSA, la ETR tiene también sus limitaciones en términos de un bajo valor predictivo positivo y especificidad $^{6-7}$. Por tanto, el principal papel de la escala de grises en la ETR es dirigir la biopsia de próstata.

La biopsia de próstata constituye la exploración esencial para el diagnóstico de cáncer prostático, permite la obtención de tejido prostático para su estudio anatomopatológico que determinará la existencia o no de células tumorales y gradación según la escala de Gleason.

Desde que se describiese la biopsia de próstata sextante hasta la actualidad, se han desarrollado distintos esquemas de biopsia ampliados a 8-12 punciones con los que se consigue un aumento en la tasa de detección de cáncer de un 8-15\% $\%^{8-9}$, reduciendo la tasa de falsos negativos en la primera biopsia de próstata. A pesar de ello, existe un porcentaje de tumores que no se diagnostican. En recientes estudios se ha descrito que entre un 10$54 \%$ de cánceres de próstata pueden pasar desapercibidos en la primera biopsia ${ }^{10-11}$ y de éstos un $11 \%$ será clínicamente significativo ${ }^{12}$.
Para la detección de estos tumores está indicada una segunda biopsia. A partir de los datos obtenidos en un estudio prospectivo sobre el cáncer de próstata (European Prostate Cancer Detection) se compararon las características de tumores diagnosticados con una y dos biopsias sin encontrarse diferencias significativas en cuanto al estadio y grado del tumor, por lo que ante la elevación del PSA y una biopsia de próstata negativa estaría indicada la rebiopsia ${ }^{13}$. Se han descrito tasas de detección de entre un $10 \%$ y $31 \%$ con esta segunda biopsia ${ }^{13-17}$.

Pero existen pacientes en los que, tras dos biopsias de próstata negativas para cáncer, hay todavía una alta sospecha de la enfermedad: persiste la elevación del PSA, o se ha diagnosticado histopatológicamente en la biopsia previa enfermedad premaligna (neoplasia intraepitelial de alto grado -HGPIN- o proliferación microacinar atípica -ASAP-).

Existe un riesgo de detección de cáncer de próstata clínicamente no significativo tras la biopsia prostática que oscila entre el 9 y el $48 \%{ }^{18}$. Otro problema que se plantea es si los tumores diagnosticados en biopsias de repetición (a partir de la tercera biopsia) son más o menos agresivos que los diagnosticados en la biopsia inicial. En un estudio en el que se compara las características histopatológicas y bioquímicas de los tumores diagnosticados en primera, segunda, tercera y cuarta biopsia se observa que los cánceres diagnosticados a partir de la tercera y cuarta biopsia tienen menor grado de Gleason, volumen y estadio que aquellos diagnosticados en primera y segunda biopsia, lo cual implicaría una menor agresividad biológica ${ }^{14}$.

Esto subraya el problema de rebiopsiar sucesivamente a pacientes que tienen un alto índice de sospecha de padecer un tumor. ¿Hasta que punto es adecuado rebiopsiar a un paciente que quizás tenga un cáncer de próstata que no le suponga una amenaza vital? El diagnóstico de un cáncer de próstata clínicamente no significativo implica un sobrediagnóstico y el consiguiente sobretratamiento en la mayoría de las ocasiones. Sin embargo, en la actualidad no disponemos de factores predictivos clínicos ni analíticos que nos ayuden a diferenciar aquellos pacientes que padecen un cáncer clínicamente significativo o no significativo. Es por ello por lo que el único factor disponible en la actualidad es el estudio histopatológico de la biopsia prostática. 


\section{OBJETIVO}

El objetivo de este trabajo es estudiar las características clínicas e histopatológicas de los tumores de próstata diagnosticados a partir de la tercera biopsia y descartar que estemos realizando un sobrediagnóstico de tumores clínicamente no significativos con la realización de más de dos biopsias de repetición.

\section{MATERIAL Y MÉTODO}

Desde Enero de 2000 a Diciembre de 2006 se han realizado en nuestro centro 2.457 biopsias de próstata transrectales a pacientes con sospecha clínica de cáncer de próstata resultando positivas para tumor en primera biopsia 810 (32,9\%). A 306 pacientes se les ha realizado una segunda biopsia de próstata cuya rentabilidad diagnóstica fue del 29,08\% (89 tumores de próstata). Se revisan las historias clínicas de 61 pacientes a los que se les ha realizado tres o más biopsias de próstata transrectales durante este periodo de tiempo.

Las indicaciones para la realización de biopsia de próstata en este subgrupo fueron la elevación persistente del PSA por encima de 4,0 ng/mL, persistencia del TR sugestivo de tumor, velocidad del PSA mayor de 0,75 ng/mL/año y hallazgo en la anatomía patológica de la biopsia previa de HGPIN o ASAP.

Empleamos un transductor endorectal EC9-4 multifrecuencia con frecuencias comprendidas entre 3.6 y $9 \mathrm{Mhz}$. Los parámetros técnicos del estudio utilizados en la ETR fueron una frecuencia de $9 \mathrm{Mhz}$ con una ganancia de 30-45 dB. En esta exploración ultrasonográfica fue valorada, en cortes transversales y longitudinales, la anatomía de la glándula prostática en términos de:

- Morfología y simetría de la misma.

- Morfología de las vesículas seminales y de los ángulos prostatoseminales.

- Integridad de la cápsula prostática y su relación con estructuras vecinas.

- Presencia de calcificaciones, quistes de retención o adenómeros en zona transicional.

- Medida del Volumen prostático y de la zona transicional.

Tras el estudio morfológico global en la ETR, se procedió a la búsqueda detenida y caracterización, lóbulo a lóbulo, de áreas hipoecoicas sospechosas de albergar células tumorales.
Cada procedimiento se realizó según el esquema de biopsia sextante clásico obteniéndose en total 6 cilindros de las zonas periféricas craneal, medial y apical; a partir del año 2004, se amplió el esquema de biopsia hasta 18 cilindros, con dos cilindros de los cuernos laterales de cada lóbulo, craneal y medial, dos cilindros de la zona transicional de cada lóbulo, craneal y medial, un cilindro del estroma fibromuscular anterior de cada lóbulo y un cilindro de la zona trapezoidal de cada lóbulo. Además se biopsió selectivamente cualquier área anómala ecográficamente, principalmente zona hipoecoicas.

Las biopsias se obtuvieron mediante una pistola de biopsia desechable con aguja de punción 18G, 25 $\mathrm{cm}$ de longitud y profundad de penetración en tejido $22 \mathrm{~mm}$, obteniendo cilindros de $1,8 \mathrm{~cm}$ de longitud. Cada cilindro de tejido prostático se depositó por separado en formol y fue remitido para su estudio al servicio de anatomía patológica.

Se utilizó como profilaxis antibiótica aminoglucósido parenteral (tobramicina $240 \mathrm{mg}$ ) pre-biopsia y una pauta de tres días de quinolona oral (ciprofloxacino 500mg). Y para el control del dolor, $50 \mathrm{mg}$ de meperidina intravenosa, o infiltración bilateral en plexo neurovascular con $5 \mathrm{ml}$ de lidocaína al $2 \%$.

En cada paciente diagnosticado de cáncer de próstata a partir de la tercera biopsia, se registró la edad, PSA, cociente PSA libre/PSA total, PSA densidad, características del TR, volumen prostático, hallazgos ecográficos sugestivos de tumor, presencia de lesión premaligna (HGPIN y/o ASAP), grado de Gleason obtenido con la biopsia de próstata y número de cilindros afectos de cáncer. De la anatomía patológica de las piezas de prostatectomía radical de los pacientes a los que se les ha realizado este tratamiento se estudió el estadio patológico según la clasificación TNM, el volumen tumoral y el grado de Gleason.

Se consideró cáncer clínicamente no significativo en la biopsia de próstata aquel que afecta a un sólo cilindro (menos del 10\% del total de la muestra obtenida) y con grado de Gleason menor de 7 , y en la pieza de prostatectomía, los tumores confinados a próstata, grado de Gleason menor de 7 y cuyo volumen es menor de 0,5 mL.

\section{RESULTADOS}

Incluimos en este estudio un total de 61 pacientes a los que se les realizaron al menos tres biopsias 
Tabla 1. Edad, próstata y valores de PSA en pacientes con cáncer de próstata.

\begin{tabular}{|c|c|c|c|}
\hline & Biopsia 3 & Biopsia 4 & Biopsia 5 \\
\hline Media edad $\pm \mathrm{DE}$ (rango) & $65,8 \pm 5,86(56-76)$ & $69,4 \pm 3,91(66-76)$ & 66 \\
\hline Media Volumen Próstata \pm DE (rango) & $56,46 \pm 18,70(23,80-87,90)$ & $33,80 \pm 2,39(30-36)$ & 54 \\
\hline Media PSA \pm DE (rango) & $19,15 \pm 28,55(5,20-120)$ & $21,78 \pm 12,99(9-38)$ & 9,2 \\
\hline Media PSA libre/PSA total \pm DE (rango) & $0,12 \pm 0,40(0,03-0,18)$ & $0,14 \pm 0,12(0,05-0,31)$ & 0,17 \\
\hline Media PSA densidad \pm DE (rango) & $0,33 \pm 0,40(0,06-1,71)$ & $0,64 \pm 0,38(0,25-1,15)$ & 0,17 \\
\hline
\end{tabular}

de próstata para diagnóstico de cáncer de próstata. A 14 de ellos se les realizaron cuatro biopsias y a dos, una quinta biopsia. En 25 pacientes se realizó la biopsia de próstata sextante clásica y en 37 se realizó según el protocolo de biopsia ampliado hasta 18 cilindros. El tiempo medio transcurrido entre biopsias es de 15,79 (2,5-74) meses.

De los 61 pacientes, 21 son diagnosticados de adenocarcinoma de próstata (porcentaje de positividad 34,42\%); 15 en su tercera biopsia, 5 en la cuarta biopsia y 1 en su quinta biopsia, siendo el porcentaje de positividad para cada subgrupo del $24,59 \%, 35,71 \%$ y $50 \%$ respectivamente.

En la Tabla 1 se detallan las características clínicas y bioquímicas (edad, volumen prostático, PSA, PSA libre/total, PSA densidad) de los pacientes diagnosticados de cáncer de próstata en la tercera, cuarta y quinta biopsia.

El tacto rectal fue sugestivo de tumor en 4 de 15 tumores diagnosticados con la tercera biopsia, y en dos de los cinco diagnosticados en la cuarta biopsia. Y los hallazgos ecográficos fueron sugestivos de tumor (áreas hipoecoicas) en 9 de 15 pacientes en los que la tercera biopsia fue positiva y en 2 de los cinco que fue positiva la cuarta biopsia. (Tablas 2 y 3).
Obtenemos, con un índice de confianza al 95\%, una sensibilidad del 28\% [11-52], especificidad del $82 \%$ [67-92] y valor predictivo positivo del 62\% [3687] para el tacto rectal como prueba diagnóstica de cáncer de próstata. En cuanto a la ecografía transrectal la sensibilidad es del 52\% [29-74], la especificidad del $85 \%$ [70-94] y el valor predictivo positivo del 77\% [60-94].

En 21 pacientes $(34,4 \%)$ la indicación de rebiopsia fue por existencia de lesión premaligna en biopsia previa. En este subgrupo el porcentaje de positividad para tumor de la rebiopsia fue del 38\%. En las Tablas 4 y 5 se presenta la asociación entre la lesión premaligna y cáncer.

Tanto el HGPIN como el ASAP resultan tests diagnósticos poco sensibles (14\% y 23\% respectivamente) para el diagnóstico de cáncer pero alcanzan una especificidad del $80 \%$ y $77 \%$ respectivamente (IC al 95\%). Los valores predictivos positivos del HGPIN y del ASAP son del 41 y del 51\% respectivamente.

De once que fueron diagnosticados de HGPIN había tumor en tres de ellos (27,2\%) y de los 14 en los que existía ASAP, cinco tenían tumor (35,7\%), sin observar diferencias estadísticamente significativas ( $\mathrm{p}=0,49$, prueba exacta de Fisher).

En la Tabla 6 se describe el índice de Gleason de adenocarcinomas diagnosticados en tercera, cuarta y quinta biopsia. En
Tabla 2. Asociación entre tacto rectal y adenocarcinoma. $P=0,22\left(X^{2}\right)$

\begin{tabular}{lccc}
\hline & Adenocarcinoma & No adenocarcinoma & Total \\
\hline T R sospechoso & 6 & 7 & 13 \\
T R no sospechoso & 15 & 33 & 48 \\
Total & 21 & 40 & 61 \\
\hline
\end{tabular}

Tabla 3. Asociación entre nódulo hipoecoico y adenocarcinoma. $\left(p=0,002, X^{2}\right)$

\begin{tabular}{lccc}
\hline & Adenocarcinoma & No adenocarcinoma & Total \\
\hline ETR sospechosa & 11 & 6 & 17 \\
ETR no sospechosa & 10 & 34 & 44 \\
Total & 21 & 40 & 61 \\
\hline
\end{tabular}
un paciente del subgrupo de tumores diagnosticados en la tercera biopsia, el material de la muestra era insuficiente para determinar el índice de Gleason.

Según los resultados de la biopsia, 6 pacientes presentaban criterios de cáncer clínicamente no significativo (28,57\%). A estos pacientes se les ofreció tratamiento expectante de su tumor, pero prefirieron tratamiento con intención curativa. 
Tabla 4. Asociación entre PIN de alto grado y adenocarcinoma ( $\mathrm{p}=0,73$, Fisher bilateral)

\begin{tabular}{lccc}
\hline & Adenocarcinoma & No adenocarcinoma & Total \\
\hline HGPIN & 11 & 6 & 17 \\
No HGPIN & 10 & 34 & 44 \\
Total & 21 & 40 & 61 \\
\hline
\end{tabular}

Tabla 5. Asociación entre ASAP y adenocarcinoma ( $p=0,57$, Fisher)

\begin{tabular}{lccc}
\hline & Adenocarcinoma & No adenocarcinoma & Total \\
\hline ASAP & 5 & 9 & 14 \\
No ASAP & 16 & 31 & 47 \\
Total & 21 & 40 & 61 \\
\hline
\end{tabular}

Tabla 6. Índice de Gleason de cánceres diagnosticados en $3^{\mathrm{a}}, 4^{\mathrm{a}}$ y $5^{\mathrm{a}}$ biopsia. En un paciente con cáncer de próstata diagnosticado en tercera biopsia no se dispuso de material histológico suficiente para establecer el grado de Gleason

\begin{tabular}{lccc}
\hline Gleason & $\mathbf{5} \mathbf{n}(\%)$ & $\mathbf{6 - 7} \mathbf{~ n ~ ( \% ) ~}$ & $>\mathbf{7} \mathbf{n}(\%)$ \\
\hline Biopsia 3 & $6(40)$ & $6(40)$ & $2(13,33)$ \\
Biopsia 4 & $2(40)$ & $2(40)$ & $1(20)$ \\
Biopsia 5 & & $1(100)$ & \\
\hline
\end{tabular}

Por tanto, a todos los pacientes diagnosticados de adenocarcinoma de próstata localizado se les aplicó tratamiento con intención curativa. A $5(23,80 \%)$ se les realizó braquiterapia, a $6(28,57 \%)$ radioterapia externa y $10(47,61 \%)$ fueron sometidos a prostatectomía radical. La pieza de prostatectomía evidenció 2 tumores de estadio pT2b, 7 tumores de estadio pT2c y un tumor de estadio pT4 por invasión del cuello vesical. Por tanto, no se encontraron cánceres de próstata clínicamente no significativos (estadio pT2a y volumen tumoral $<0,5 \mathrm{cc}$ ) en aquellos pacientes sometidos a cirugía radical.

En la Tabla 7 se detallan las características histopatológicas de los cánceres de próstata diagnosticados (Gleason de la biopsia, número de cilindros afectos, Gleason de la pieza de prostatectomía).

\section{DISCUSIÓN}

En nuestra serie, a diferencia de los datos descritos en la literatura revisada, existe una tendencia creciente en cuanto a la rentabilidad diagnóstica de las biopsias de repetición (Tabla 8).

Hasta Diciembre de 2004 se realizaron las biopsias de próstata según el esquema sextante clásico tanto en primera biopsia como en biopsias de repetición. Posteriormente y de acuerdo con la tendencia generalizada a la realización de biopsias ampliadas con las que se aumenta el porcentaje de detección de cáncer ${ }^{8-9}$, se realizaron con toma de mayor número de cilindros.

De los 14 cánceres de próstata diagnosticados en la tercera biopsia, nueve de ellos (64,2\%) lo fueron a partir de 2004 con protocolos de biopsia ampliados. Así pues, se observa que con la biopsia de próstata sextante se infradiagnosticaron tumores que fueron detectados tras la realización de la biopsia con toma

Tabla 7. Características histopatológicas del cáncer de próstata.

\begin{tabular}{lcc}
\hline & Biopsia 3 & Biopsia 4 \\
\hline Media Gleason biopsia \pm DE (rango) & $5,46 \pm 1,80(2-10)$ & $5,80 \pm 1,92(3-8)$ \\
Media Cilindros afectos \pm DE (rango) & $2,28 \pm 1,54(1-6)$ & $3 \pm 1,87(1-6)$ \\
Media Gleason prostatectomía DE (rango) & $6,20 \pm 0,83(5-7)$ & $5,67 \pm 1,15(5-7)$ \\
\hline
\end{tabular}

Tabla 8. Porcentaje de positividad de la primera, segunda, tercera, cuarta y quinta biopsia de próstata.

\begin{tabular}{|c|c|c|c|c|c|}
\hline Referencia & $\begin{array}{c}\text { Primera } \\
\text { biopsia (\%) }\end{array}$ & $\begin{array}{c}\text { Segunda } \\
\text { biopsia (\%) }\end{array}$ & $\begin{array}{c}\text { Tercera } \\
\text { biopsia (\%) }\end{array}$ & $\begin{array}{c}\text { Cuarta } \\
\text { biopsia (\%) }\end{array}$ & $\begin{array}{c}\text { Quinta } \\
\text { biopsia (\%) }\end{array}$ \\
\hline Djavan et al. ${ }^{14}$ & 22 & 10 & 5 & 4 & - \\
\hline Durkan et al. ${ }^{11}$ & - & 22,4 & 8,16 & - & - \\
\hline Lopez-Corona et al. ${ }^{16}$ & 77 & 17 & 4 & 2 & 0 \\
\hline Luján et al. ${ }^{17}$ & 14,4 & 15,7 & 9,8 & - & - \\
\hline Hospital La Fe & 32,9 & 24,05 & 24,59 & 35,71 & 50 \\
\hline
\end{tabular}


de mayor número de cilindros aumentando por tanto el porcentaje de positividad en estas biopsias respecto a las realizadas según el esquema sextante clásico.

En cuanto a la rentabilidad diagnóstica en cuarta y quinta biopsia, hay que destacar además que se realizan a pacientes seleccionados con alta sospecha de cáncer de próstata (elevación persistente del PSA por encima de $4.0 \mathrm{ng} / \mathrm{mL}$, persistencia del TR sugestivo de tumor, velocidad del PSA mayor de 0,75 ng/mL/año y hallazgo en la anatomía patológica de la biopsia previa de HGPIN o ASAP) por lo que la probabilidad de diagnóstico de cáncer de próstata es mayor.

Otro de los factores que podrían haber contribuido a la alta tasa de detección en las biopsias de repetición es la realización de éstas a pacientes con existencia de lesión premaligna en biopsias previas, pero en nuestra serie el riesgo de cáncer en la rebiopsia tras el diagnóstico de PIN de alto grado y ASAP (27,2\% y 35,7\% respectivamente) es similar al descrito en la literatura, por tanto no es este subgrupo de biopsias, cuya indicación fue la lesión premaligna, las responsables de la tasa diagnóstica elevada. Epstein et al., en un artículo de revisión sobre lesión premaligna en biopsia de próstata, describe un riesgo medio de cáncer del $24,1 \%$ tras el diagnóstico de PIN de alto grado y del $40 \%$ después de ASAP $^{19}$.

El diagnóstico de lesión premaligna en biopsias de próstata y su manejo es otro de los puntos sobre el cual todavía no existe consenso. Es importante distinguir entre HGPIN y ASAP ya que estas dos entidades se corresponden con hallazgos histológicos diferentes que tienen distinta probabilidad de diagnóstico de cáncer en la rebiopsia. La existencia de glándulas atípicas sospechosas de carcinoma se asocia con un mayor riesgo de cáncer en la rebiopsia comparado con PIN de alto grado ${ }^{19}$ tal y como hemos observado en este trabajo.

La realización de biopsias de repetición y ampliadas a mayor número de cilindros ha supuesto un aumento en el porcentaje de detección de cánceres de próstata aparentemente no significativos. A menudo nos encontramos con pacientes que presentan sospecha clínica de cáncer de próstata y en la biopsia se identifica un único foco diminuto de adenocarcinoma microacinar prostático. Resulta en estos casos difícil la elección del tratamiento más adecuado, sobretodo si consideramos el riesgo de someter al paciente a un tratamiento radical, con su morbilidad asociada, para tratamiento de una enfermedad clínicamente insignificante.

La posibilidad de diagnóstico de enfermedad clínicamente no significativa de entre el 9 y $50 \%{ }^{18,20}$ con el consiguiente sobretratamiento. En nuestra serie la realización de la tercera, cuarta y quinta biopsia ha supuesto el sobrediagnóstico en el $28,57 \%$ de los pacientes.

Pero no debemos olvidar que, hasta un $28 \%$ de varones con un valor de PSA $<4 \mathrm{ng} / \mathrm{ml}$ tendrán cáncer de próstata y de éste grupo, hasta un 25\% se corresponde con enfermedad de alto grado ${ }^{21,22}$. Por tanto, existen cánceres prostáticos de diferente comportamiento biológico para los que, hasta el momento, no hemos encontrado factores predictivos de su agresividad. Son necesarios marcadores de mayor especificidad que el PSA sérico y sus variantes para poder diferenciar aquellos pacientes en los que el cribaje de la enfermedad supondría un sobrediagnóstico de la misma de aquellos que tienen latente un adenocarcinoma de próstata que sería clínicamente significativo.

\section{CONCLUSIÓN}

En nuestro medio, la tercera, cuarta y quinta biopsia alcanza una positividad del $34,42 \%$ y se corresponde con tumores que en hasta un $71,42 \%$ son clínicamente significativos y se benefician de un tratamiento con intención curativa.

\section{REFERENCIAS}

1. http://www.ine.es/jaxi/tabla.do. 1 de septiembre de 2008.

2. Carvalhal GF, Smith DS, Mager DE, Catalona WJ. Digital rectal examination for detecting prostate cancer at prostate specific antigen levels of $4 \mathrm{ng} / \mathrm{ml}$ or less. J Urol. 1999;161:835-839.

3. Eastham JA, May R, Robertson JL, Sartor O, Kattan MW. Development of a nomogram that predicts the probability of a positive prostate biopsy in men with an abnormal digital rectal examination and a prostate-specific antigen between 0 and 4 ng/ml. Urology. 1999;54:708-713.

4. Catalona WJ, Richie JP, Ahmann FR, Hudson MA, Scardino PT, Flanigan RC, et al. Comparison of digital rectal examination and serum prostate specific antigen (PSA) in the early detection of prostate cancer: results of a multicentre clinical trial of 6,630 men. J Urol. 1994;151:1283-1290.

5. Lodding P, Aus G, Bergdahl S, Frosing R, Lilja H, Pihl CG, et al. Characteristics of screening detected prostate cancer in men 50 to 66 years old with 3 to $4 \mathrm{ng} / \mathrm{mL}$. Prostate specific antigen. J Urol. 1998;159:899-903.

6. Alexander AA. To color doppler image the prostate or not: that is the question. Radiology. 1995;195:11-14. 
7. Kelly IM, Lees WR, Rickards D. Prostate cancer and the role of color doppler US. Radiology 1993;189:153-159.

8. Norberg M, Egevad L, Holmberg L, Sparen P, Norlen BJ, Busch C. The sextant protocol for ultrasound-guided core biopsy cores of the prostate understimates the presence of cancer. Urology. 1997;50:562-568.

9. Garber SJ, Goldenberg SL, Cooperberg PL, Wong Ad, Bilby JH, Mathieson JR. Systematic transrectal ultrasound-guided biopsy of the prostate. Can Assoc Radiol J. 1994;45:387-400.

10. Patel AR, Jones JS, Rabets J, DeOreo G, Zippe CD. Parasagittal biopsies add minimal information in repeat saturation prostate biopsy. Urology. 2004;63:87-96.

11. Durkan GC, Greene DR. Elevated serum prostate specific antigen levels in conjuntion with an initial prostatic biopsy negative for carcinoma: who should undergo a repeat biopsy? BJU Int. 1999;83:34-42.

12. Park PC, Mai T, Roustan Delatour N, Morash C, Cagiannos I. Predictive value of prostatic adenocarcinoma after a negative prostate biopsy. BJU Int. 2006;98:986-988.

13. Djavan B, Milani S, Remzi M. Prostate biopsy: who, how and when. An update. Can J Urol. 2005; 12:44-52.

14. Djavan B, Ravery V, Zlotta A, Dobronski P, Dobrovits M, Fakhari $\mathrm{M}$, et al. Prospective evaluation of prostate cancer detected on biopsies 1, 2, 3 and 4: when should we stop? J Urol. 2001; 166:1679-1683.

15. Yuen JS, Lau WK, Ng LG, Tan PH, Khin LW, Cheng CW. Clinical, biochemical and pathological features of initial and repeat transrectal ultrasonography prostate biopsy positive patients. Int $J$ Urol. 2004;11:225-231.

16. Lopez-Corona E, Ohori M, Wheeler TM, Reuter VE, Scardino PT, Kattan MW, et al. Prostate cancer diagnosed after repeat biopsies have a favorable pathological outcome but similar recurrence rate. J Urol. 2006;175:923-930.
17. Lujan M, Paez A, Santonja C, Pascual T, Fernandez I, Berenguer A. Prostate cancer detection and tumor characteristics in men with multiple biopsy sessions. Prostate 2004;7: 238-242.

18. Taverna G, Colombo P, Seveso M, Giusti G, Piccinelli A, Benetti A, et al. Single small focus of prostate adenocarcinoma $(<$ or $=$ $1 \mathrm{~mm}$ and too small for grading) and clinical significant disease after radical prostatectomy. Arch Ital Urol Androl. 2006;78:5760.

19. Epstein JI, Herawi M. Prostate Needle Biopsies Containing Prstatic Intraepithelial Neoplasia or Atypical Foci Suspicious for Carcinom: Implications for Patient Care. J Urol. 2005;175: 820-834.

20. Draisma G, Boer R, Otto SJ, van der Cruijsen IW, Damhuis RA, Schroder FH, et al. Lead times and overdetection due to prostate specific antigen screening: estimates from the European Randomized Study of Screening for Prostate Cancer. J Natl Cancer Inst. 2003;95: 868-878.

21. Thompson IM, Pauler DK, Goodman PJ. Prevalence of prostate cancer among men with a prostate specific antigen level $\leq 4 \mathrm{ng}$ per milliliter. N Eng J Med. 2004;350:2239-2246.

22. Abdelhady M, Abusamra A, Pautler SE, Chin JL, Izawa JI. Cliniclly significant prostate cancer found incidentallly in radical cystoprostatectomy specimens. BJU Int. 2006;99:326-329.

Correspondencia autor: Dra. M.P. Bahílo Mateu Servicio de Urología. Hospital Universitario La Fe Avda. Campanar, 21- 46009. Valencia. Tel.: 963862700 E-mail autor: pilarbahilo@comv.es

Información artículo: Original - Cáncer de próstata

Trabajo recibido: abril 2008

Trabajo aceptado: julio 2008 